Draft Version September 10, 2018

Preprint typeset using $\mathrm{I}_{\mathrm{A}}^{\mathrm{T}} \mathrm{EX}$ style emulateapj v. 10/10/03

\title{
ASYMMETRIES IN THE COSMIC MICROWAVE BACKGROUND ANISOTROPY FIELD
}

\author{
H. K. ERIKSEN ${ }^{1}$ \\ Institute of Theoretical Astrophysics, University of Oslo, P.O. Box 1029 Blindern, \\ N-0315 Oslo, Norway \\ F. K. HANSEN \\ Dipartimento di Fisica, Università di Roma 'Tor Vergata', Via della Ricerca Scientifica 1, I-00133 Roma, Italy
}

A. J. BANDAY

Max-Planck-Institut für Astrophysik, Karl-Schwarzschild-Str. 1, Postfach 1317, D-85741 Garching bei München, Germany

\author{
K. M. GóRSKI ${ }^{2}$ \\ Jet Propulsion Laboratory, MS 169/327, 4800 Oak Grove Drive, Pasadena CA 91109 \\ AND \\ P. B. LILJE ${ }^{1}$ \\ Institute of Theoretical Astrophysics, University of Oslo, P.O. Box 1029 Blindern, \\ N-0315 Oslo, Norway \\ (Dated: Received 2003 July 30 / Accepted 2003 December 29) \\ Draft version September 10, 2018
}

\begin{abstract}
We report on the results from two independent but complementary statistical analyses of the WMAP first-year data, based on the power spectrum and $N$-point correlation functions. We focus on large and intermediate scales (larger than about $3^{\circ}$ ) and compare the observed data against Monte Carlo ensembles with WMAP-like properties. In both analyses, we measure the amplitudes of the large-scale fluctuations on opposing hemispheres and study the ratio of the two amplitudes. The power-spectrum analysis shows that this ratio for $W M A P$, as measured along the axis of maximum asymmetry, is high at the 95\%-99\% level (depending on the particular multipole range included). The axis of maximum asymmetry of the WMAP data is weakly dependent on the multipole range under consideration but tends to lie close to the ecliptic axis. In the $N$-point correlation function analysis we focus on the northern and southern hemispheres defined in ecliptic coordinates, and we find that the ratio of the large-scale fluctuation amplitudes is high at the 98\%-99\% level. Furthermore, the results are stable with respect to choice of Galactic cut and also with respect to frequency band. A similar asymmetry is found in the $C O B E$-DMR map, and the axis of maximum asymmetry is close to the one found in the WMAP data.

Subject headings: cosmic microwave background - cosmology: observations - methods: statistical
\end{abstract}

\section{INTRODUCTION}

In recent months much interest has focused on the question of the lack of large-scale power in the $W M A P^{3}$ (Bennett et al. 2003a) first-year data sets (e.g., Efstathiou 2003a, b; Efstathiou 2004; Spergel et al. 2003; Tegmark, de Oliveira-Costa, \& Hamilton 2003). Evidence for non-Gaussian features in the sky maps has also been claimed (Coles et al. 2004; Naselsky et al. 2004; Vielva et al. 2004; Copi, Huterer, \& Starkman 2004). In this paper we present evidence of a related effect, namely that the large-scale power in $W M A P$ is unevenly dis-

\footnotetext{
${ }^{1}$ Also at Centre of Mathematics for Applications, University of Oslo, P.O. Box 1053 Blindern, N-0316 Oslo, Norway

Electronic address: h.k.k.eriksen@astro.uio.no

Electronic address: Frode.Hansen@roma2.infn.it

Electronic address: banday@MPA-Garching.MPG.DE

2 Also at Warsaw University Observatory, Aleje Ujazdowskie 4, 00-478 Warszawa, Poland

Electronic address: Krzysztof.M.Gorski@jpl.nasa.gov

Electronic address: per.lilje@astro.uio.no

${ }^{3}$ Wilkinson Microwave Anisotropy Probe
}

tributed on the sky. This effect can be seen on angular scales down to $3-5^{\circ}$, or $\ell \lesssim 40$. Similar results based on the genus statistic have also been reported by Park (2004).

\section{DATA AND SIMULATIONS}

All results in this paper are derived from the WMAP first year Q-, V- and W-band sky maps, corrected for foreground emission using the template method of Bennett et al. (2003b). The power spectrum analysis includes the co-added V-and W-band information, while the $N$-point correlation function analysis utilizes the Q-, $\mathrm{V}$ - and $\mathrm{W}$-band data both separately and in a co-added form. The maps are combined using inverse-variance noise weights as described by Hinshaw et al. (2003b).

The significance of our analyses are evaluated by means of Monte Carlo simulations: we generate an ensemble of stochastic realizations of the CMB sky to which the observed data are compared through the power spectrum and $N$-point statistics. The CMB simulations are based on the WMAP running-index spectrum. From 
each $\mathrm{CMB}$ realization, we generate simulated skies corresponding to the eight WMAP Q-, V-and W-band sky maps by convolution with the appropriate beam transfer functions (Page et al. 2003) and then adding a corresponding noise realization, based on the specific noise properties of the channel in question.

\section{ANALYSIS METHODS}

In this paper we analyze the WMAP data by means of both the angular power spectrum (Hansen, Górski, \& Hivon 2002) and its real-space complements, the $N$-point correlation functions (e.g., Eriksen, Banday, \& Górski 2002). While we do not aim at presenting the full statistical machinery behind these methods here, instead referring the interested reader to the above papers for details, we do provide the specifics appropriate for the WMAP assessment.

\subsection{The angular power spectrum}

The angular power spectrum can be determined using a maximum likelihood inference from a local estimate on a given patch of the sky, assuming that the coupling between harmonic modes due to the incomplete sky coverage can be evaluated. Hansen et al. (2002) have demonstrated that for an axisymmetric patch, this correlation matrix can be computed analytically. In this paper, we utilize a Monte Carlo approach to calculate the matrix on a general nonsymmetric sky patch.

Using this likelihood technique, we have estimated the power spectrum on 164 slightly overlapping discs with radius $9: 5$ uniformly distributed on the part of the sphere outside of the WMAP Kp2 sky cut. In particular, we have compared the disc spectra in the northern Galactic hemisphere to those in the southern sky for the multipole bin $\ell=2-63$.

We extend the method further by estimating the power spectra directly for hemispheres, thus allowing us to obtain a higher multipole resolution on large scales (low- $\ell$ ). In order to avoid the introduction of bias through consideration of hemispheres defined only with respect to the Galactic coordinate system, we estimated the spectra for a number of hemispheres defined by orienting the north-south axis in 82 different directions on the sphere. Specifically, we applied the following procedure both for the WMAP co-added V- and W-bands, plus each of 2048 simulations.

- The power spectrum was estimated in bins $C_{b}$ of width 3 , where the multipole bin $b \in\left\{\ell_{\min }^{b}, \ell_{\max }^{b}\right\}$ is defined by $\ell_{\min }=2+3 b$ and $\ell_{\max }=4+3 b$. The spectrum was successively estimated on the northern and southern hemispheres with the axis defining north and south oriented in 82 different directions.

- The estimated bins $C_{b}$ in a given multipole range $\ell=\ell_{\min }-\ell_{\max }$ were summed for each of the 164 hemispheres giving $C_{\text {hemisphere }}=\sum_{b} C_{b}$. The ratio between the northern and southern spectra for each of the 82 possible orientations $r=$ $\max \left(C_{N} / C_{S}, C_{S} / C_{N}\right)$ was determined.

- The axis with the maximum asymmetry ratio, $r_{\max }$, was recorded.
Subsequently, histograms of the asymmetry ratio were used to quantify the level of agreement between the simulations and the real sky measurements. The results are presented in 4.1 .

\section{2. $N$-point correlation functions}

An $N$-point correlation function is determined from the average product of $N$ temperatures, as measured in a fixed relative orientation defined by an $N$-point polygon on the sky. Algorithmically speaking, these functions are estimated as simple averages over all sets of pixels fulfilling the geometric requirements set by the polygon.

In this paper, the correlation function analyses have been designed to probe two different ranges of scales.

The very largest scales are analyzed by measuring the three- and four-point correlation functions from the full sky in $1^{\circ}$ bins, for a total of 120 (equilateral and pseudocollapsed) ${ }^{4}$ three-point configurations and 235 (rhombic, 2+2-point and 1+3-point; see Eriksen et al. 2002) four-point configurations. To facilitate this analysis, the large-scale $N$-point correlation functions are computed from low-resolution maps, which are constructed by first de-convolving the initial WMAP beam transfer function then convolving with a Gaussian beam of $\mathrm{FWHM}=140^{\prime}$. The maps, initially at a HEALPix ${ }^{5}$ resolution of $N_{\text {side }}=$ 512 , are then reconstructed at $N_{\text {side }}=64$, applying suitable corrections for the pixel window functions at the two resolutions. A best-fit monopole, dipole and quadrupole are subtracted from the sky maps before the $N$-point correlation functions are determined on that part of the sky defined by the WMAP Kp0 mask. However, since our processing smooths the maps with a relatively wide Gaussian beam, residual, inadequately corrected foreground structure in the Galactic plane could also be smoothed beyond the boundaries of the standard Kp0 mask. For this reason, we extend the mask accordingly in all directions before proceeding. This extended mask no longer includes the point source exclusion regions since the smoothing process minimizes their contribution to our analysis.

The motivation for removing the quadrupole is simply that the observed quadrupole amplitude is apparently low in the WMAP data, and whether this is a real feature or simply the outcome of inadequate foreground removal remains unresolved (see, e.g., Efstathiou 2003b; Efstathiou 2004). Since correlation functions are intrinsically more sensitive to low- $\ell$ multipoles and it is difficult to include the uncertainties related to foreground removal, we choose to eliminate any concern that this effect could dominate other interesting results.

The two-point correlation function is also evaluated but it has proved difficult to define an asymmetry parameter in this case, and the results are therefore omitted in this paper. Nevertheless, the four-point functions are compared against the value inferred by the two-point function of the same sky, as described by Eriksen et al. (2002).

Intermediate scales between 3 and $5^{\circ}$ are probed by partitioning the sphere into 81 discs, each of $10^{\circ}$ radius,

\footnotetext{
4 A "pseudo-collapsed" triangle is in this paper defined as an isosceles triangle with a baseline between 1 and $2^{\circ}$; see Gaztañaga \& Wagg (2003).

${ }^{5}$ http://www.eso.org/science/healpix/
} 

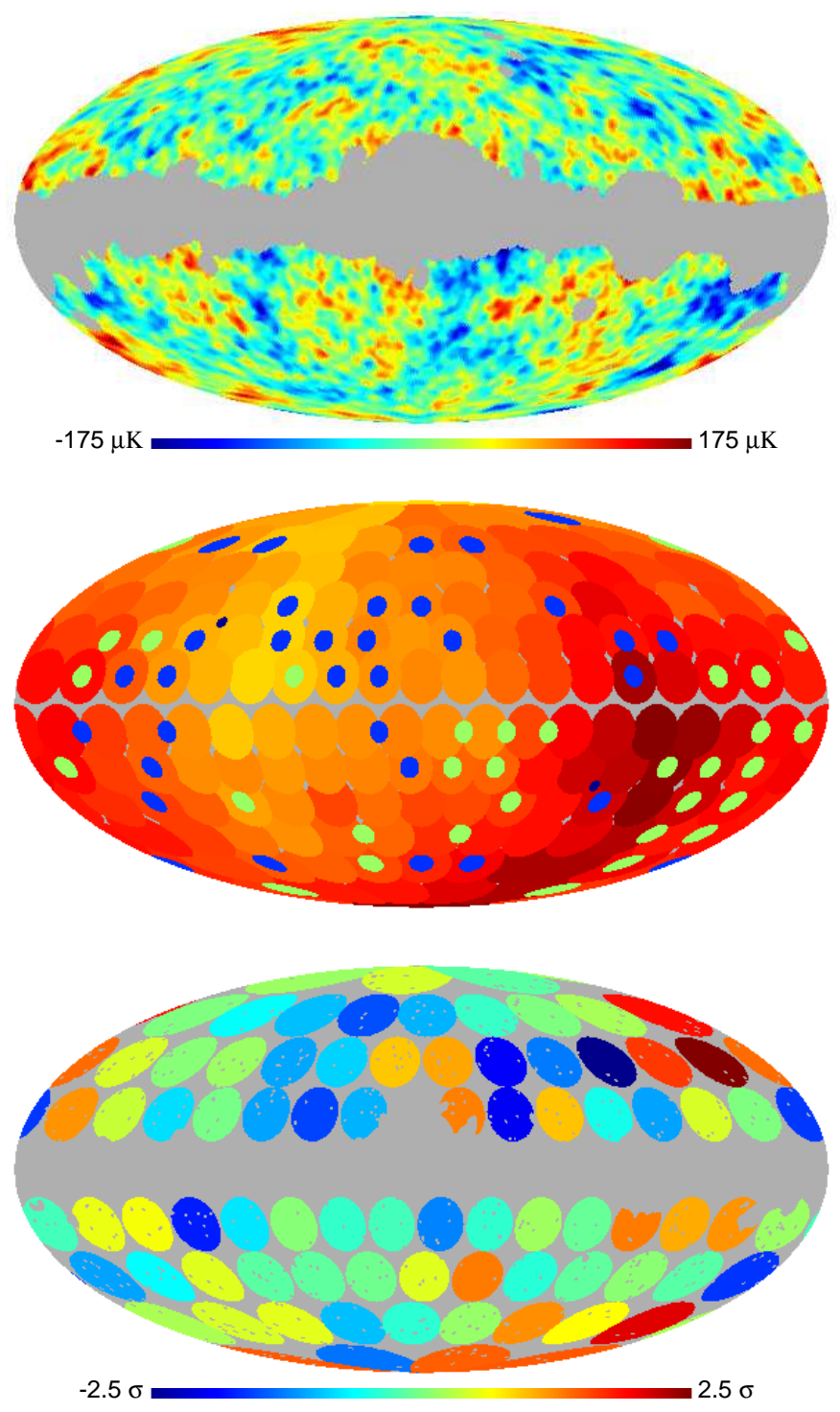

FIG. 1.- Top panel: The low-resolution Q-, V- and W-band co-added WMAP map, to which the extended Kp0 mask has been applied.

Middle panel: Results from the local power spectrum analysis. The color of the large discs indicate the ratio between the $\ell=2-63$ power spectrum bin of the northern and southern hemispheres, as determined in a reference frame where the north pole pierces the center of the disc; light red/yellow indicates a low ratio, dark red a high ratio. The medium sized dots indicate the absolute value of the power spectrum estimated on a 9.5 disc in the $\ell=2-63$ bin (i.e., not on the full hemisphere), and a dark blue dot means that this value lies below the lower $80 \%$ confidence limit, while a green dot means it lies above the upper $80 \%$ limit. Finally, the ecliptic poles are marked by the small dark-blue spots. The figure should not be interpreted as implying that data close to the galactic plane have been used in the analysis: the WMAP Kp2 mask has been applied for all power spectrum computations.

Bottom panel: The results from the intermediate scale three-point analysis. Blue corresponds to low $\chi^{2}$, which again corresponds to small fluctuations in the three-point function, while red corresponds to high $\chi^{2}$. The full Kp0 mask has been applied to the data. and computing the three-point correlation function for each disc. The full Kp0 mask is applied to the sky map, including the regions of exclusion related to known point sources. In order to reduce disc-disc correlations we first apply a high-pass filter to the maps, removing all multipoles with $\ell=0, \ldots, 18$. On each of these discs, we compute the three-point correlation functions for 460 isosceles configurations smaller than $5^{\circ}$.

The degree of agreement between the simulations and the observations are quantified in terms of a standard covariance matrix $\chi^{2}$ statistic. Such a statistic is, in principle, only appropriate if the data under consideration follow a joint Gaussian distribution. Usually, it also works quite well for mildly non-Gaussian distributions, and in particular symmetric ones, but for strongly asymmetric distributions it is likely to yield biased results. It is easily seen that the distributions for the even-ordered correlation functions for a given geometrical configuration are in general strongly asymmetric. We therefore transform the data of each configuration into a Gaussian distribution by means of the empirical distribution function, before performing the $\chi^{2}$ analysis. The transformation is defined as follows

$$
\frac{\text { Rank of observed map }}{\text { Total number of maps }+1}=\frac{1}{\sqrt{2 \pi}} \int_{-\infty}^{s} e^{-\frac{1}{2} t^{2}} d t \text {. }
$$

The left-hand side is the fraction of simulations with a lower correlation function value than the map under consideration (i.e., it approximates the true, but unknown, cumulative distribution function), and the right-hand side yields the corresponding value, $s$, measured relative to a standard normal distribution.

\section{RESULTS}

\subsection{Power spectrum}

We have computed a local power spectrum estimate for 164 slightly overlapping discs with radius 9.5 , uniformly distributed on the part of the sphere outside of the WMAP Kp2 sky cut and compared these to spectra derived from an ensemble of 6144 simulated maps. Concentrating on the lowest multipole bin $\ell=2-63$ we found that the amplitudes for discs in the northern Galactic hemisphere were generally lower in the WMAP data than in the simulated maps. Conversely, we found that the discs in the southern Galactic hemisphere were of generally higher amplitude than in the simulations. By considering the ratio of the mean of the spectra in the northern hemisphere to that in the southern hemisphere, we found that only $0.5 \%$ of the simulations have a ratio as low as the WMAP data. This is the first evidence of a large scale absence of power in one hemisphere of the WMAP data.

In order to pursue this effect further, we have computed the ratio of the power spectrum amplitudes determined for the northern and southern hemispheres as defined in a particular coordinate system, and for a selection of multipole ranges. The results are reported in Table 1] In particular, we consider this ratio after first determining that coordinate frame that maximizes its value. The ratio for the $W M A P$ data is larger than $\sim 99 \%$ of the maximum asymmetry values determined from the simulated maps. We have also tabulated the orientation, in Galactic coordinates, of the north pole for the data-preferred reference frame. Note that whereas 
TABle 1. PoWer SPECTRUM ASYMmetry RATIO RESUlts

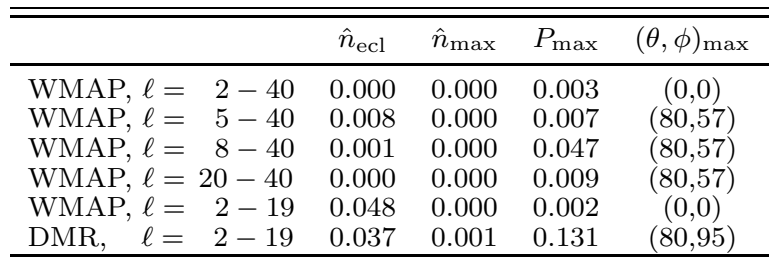

Note. - A summary of results for the ratio of power spectrum amplitudes between the northern and southern hemispheres over various $\ell$-ranges defined over the WMAP coadded V-and W-band data after applying the Kp2 mask. First column: The ratio as computed in the ecliptic coordinate frame. The numbers indicate the fraction of simulations with a higher asymmetry ratio for the ecliptic axis than the data.

Second column: The ratio as computed in a coordinate frame selected such that the observed asymmetry is maximized for the observational data alone. The data values are compared against values from the simulations that have the preferred axis imposed on them by the data. The numbers indicate the fraction of simulations with a higher asymmetry ratio for this axis than the data.

Third column: The ratio as computed in a coordinate frame selected such that the observed asymmetry is maximized. The numbers indicate the fraction of simulations with a higher maximum ratio $r$ than that found in the $W M A P$ or $C O B E$ DMR data. Note that in this case the data value is compared against values derived from the simulations that may or may not have the same preferred axis as the data.

Fourth column: The $(\theta, \phi)$ direction of the north pole in the galactic reference frame for the axis that maximizes the asymmetry observed in the data.

the asymmetry on the lowest multipoles seems to be concentrated about the north Galactic pole, the asymmetry in the higher multipoles $(5<\ell<40)$ seems to be highest about the axis with the north pole at $(\theta, \phi)=\left(80^{\circ}, 57^{\circ}\right)$ in Galactic coordinates. ${ }^{6}$ Such a result may argue against an explanation in terms of residual foreground contamination, at least for the higher multipole ranges. It is also clear that the observed asymmetry is not simply a reflection of the possible low quadrupole and octopole amplitudes found by the WMAP team. The middle panel of Figure 1 summarizes these results in a different way. Each observed disc on the map represents the statistical deviation, as compared to simulations of the observed asymmetry ratio when computed in the reference frame for which the north pole pierces the center of the disc. What is most immediately evident is that there is an apparent lack of large-scale power in the vicinity of the north ecliptic pole.

Figure 2 compares the nearly full-sky power spectrum computed by the WMAP team to the local northern and southern hemisphere estimates derived in the reference frame that maximizes the asymmetry between them for the multipole range $\ell=5-40$. This figure also shows the best-fit running-index spectrum. We see that the northern spectrum is systematically lower than the southern spectrum over almost the entire multipole range.

${ }^{6}$ Here, $\theta$ and $\phi$ are measured in the HEALPix convention, thus corresponding to co-latitude and longitude.

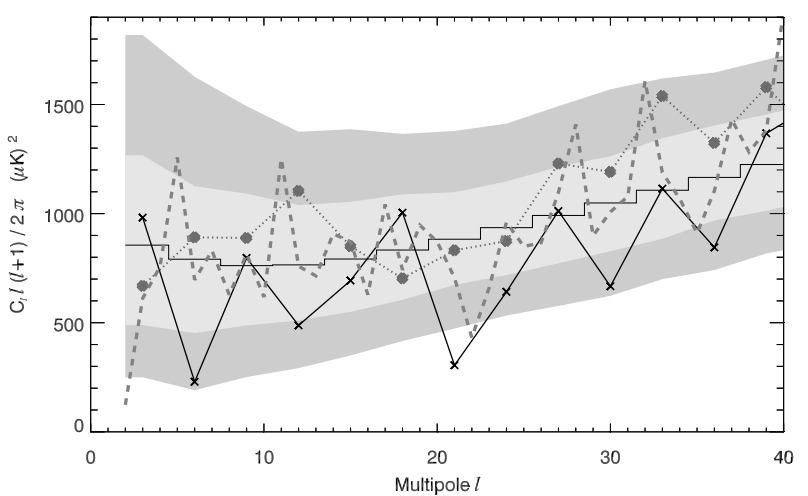

Fig. 2.- Power spectra computed from the co-added V-and Wband WMAP data. The solid line (histogram) indicates the theoretical best-fit $W M A P$ running index power spectrum. The dashed line shows the estimated power spectrum obtained by the WMAP team for the Kp2 mask. The black crosses and gray dots represent our estimates of the power spectra on the northern and southern hemispheres, respectively. Here, north and south are defined with respect to the axis that maximizes the asymmetry in the WMAP data for the corresponding hemispheres, such that the north pole is located at $(\theta, \phi)=\left(80^{\circ}, 57^{\circ}\right)$. The gray bands indicate the 1 and $2 \sigma$ confidence regions, as computed from the ensemble of 2048 Monte Carlo simulations. Formally, these error bounds differ between the hemispheres, but in practice, the difference is small and only the values from the northern hemisphere are shown.

As a useful cross check that should help to mitigate against systematic effects as the cause of the observed structure, the same hemisphere exercise has been performed for the co-added $53+90 \mathrm{GHz} C O B E$-DMR map, for which we consider multipoles in the range $\ell=2-19$ where the signal is dominant. We find that the DMR axis of maximum asymmetry lies close to that for the WMAP data. However, for DMR the significance of the result is lower at about $87 \%$ confidence. Nevertheless, given the noisier nature of the data, we consider that this is supportive of the asymmetry result.

\section{2. $\quad N$-point correlation functions}

In Figure 3, the pseudo-collapsed three-point and $1+3$ four-point functions are shown as computed for the northern and southern ecliptic hemispheres. While the expectation values of the two functions are very different, the observed behavior of the two functions is internally consistent: the northern hemisphere correlation functions are strikingly featureless (the three-point function lies very close to zero, and the four-point function drops off very quickly), while the southern hemisphere functions show relatively strong fluctuations.

In order to quantify these statements, we use the full covariance matrix $\chi^{2}$ statistic including all bin-to-bin correlations. The results from these computations are shown in Table 2 The first two rows for each mask (Kp0 and $\mathrm{Kp} 0+|b|>30 \mathrm{deg}$ ) indicate the frequency of simulations with a lower $\chi^{2}$ value than the WMAP data, and the third row shows the frequency of simulations with a smaller $\chi_{\text {north }}^{2} / \chi_{\text {south }}^{2}$. This latter statistic merits some explanation. The $\chi^{2}$ statistic in itself measures the overall consistency of an observed function with a predefined model relative to the standard deviation of the model. Thus, for a function with vanishing mean (such as the 
TAble 2. $N$-Point Correlation function $\chi^{2}$ Results

\begin{tabular}{|c|c|c|c|c|c|}
\hline & Mask & Q & V & W & Co-added \\
\hline \multicolumn{6}{|c|}{ Three-point function } \\
\hline Northern & Kp0 & 0.047 & 0.023 & 0.014 & 0.034 \\
\hline Southern & Kp0 & 0.831 & 0.861 & 0.871 & 0.840 \\
\hline Ratio of $\chi^{2}$ 's & Kp0 & 0.031 & 0.014 & 0.012 & 0.023 \\
\hline Northern & $|b|>30^{\circ}$ & 0.046 & 0.033 & 0.038 & 0.041 \\
\hline Southern & $|b|>30^{\circ}$ & 0.792 & 0.820 & 0.829 & 0.793 \\
\hline Ratio of $\chi^{2}$ 's & $|b|>30^{\circ}$ & 0.040 & 0.031 & 0.031 & 0.039 \\
\hline \multicolumn{6}{|c|}{ Four-point function } \\
\hline Northern & Kp0 & 0.070 & 0.054 & 0.050 & 0.058 \\
\hline Southern & Kp0 & 0.852 & 0.873 & 0.846 & 0.857 \\
\hline Ratio of $\chi^{2}$ 's & Kp0 & 0.030 & 0.021 & 0.025 & 0.025 \\
\hline Northern & $|b|>30^{\circ}$ & 0.020 & 0.010 & 0.012 & 0.014 \\
\hline Southern & $|b|>30^{\circ}$ & 0.927 & 0.942 & 0.931 & 0.932 \\
\hline Ratio of $\chi^{2}$ 's & $|b|>30^{\circ}$ & 0.004 & 0.001 & 0.002 & 0.002 \\
\hline
\end{tabular}

Note. - Results from $\chi^{2}$ tests of the large-scale correlation functions computed from the northern and southern ecliptic hemispheres and from the ratio of northern to southern $\chi^{2}$ for the given bands. Reported here are the frequencies of simulated realizations with a $\chi^{2}$ value $\left(\chi^{2}\right.$ ratio) lower (smaller) than for the WMAP map. The $|b|>30^{\circ}$ constraint is enforced in addition to the $\mathrm{Kp} 0$ mask.

three-point and the reduced four-point functions) the $\chi^{2}$ statistic is a monotonic function of the overall fluctuation level. Therefore, we choose the $\chi^{2}$ itself as a measure of the fluctuation amplitudes, and quantify the degree of asymmetry between the two hemispheres by a parameter that is then the ratio of the two $\chi^{2}$ values.

The conclusions to be drawn from Table 2 are as follows. The northern ecliptic hemisphere shows a consistently low level of fluctuations, independent of frequency and Galactic cut, but inside the allowed range set by the model sky. The southern hemisphere shows a slightly high level of fluctuations but is again acceptable given the model. However, the two are only marginally consistent internally: as measured by the three-point function only $\sim 2 \%$ of the simulations have a smaller ratio of $\chi^{2}$ with the Kp0 mask, and $\sim 4 \%$ with the additional constraint that $|b|>30^{\circ}$. Somewhat stronger numbers are found by the four-point function. Again, about $2 \%$ of the simulations have a smaller ratio of $\chi^{2}$ in the $\mathrm{Kp} 0$ region, but this time only $0.1 \%-0.4 \%$ have a smaller ratio in the extended region. The latter values tend to refute the possibility that residual foregrounds could be responsible for the observed asymmetry. Note also that the strongest results are found in the bands least affected by foregrounds, $\mathrm{V}$ and $\mathrm{W}$.

The top panel of Figure 1 shows the smoothed, coadded WMAP map, and in this figure it is actually possible to see the asymmetry by eye; while there are conspicuous large-scale structures visible on the southern hemisphere, the northern hemisphere is surprisingly featureless. Therefore, in order to localize these features further, we have computed the three-point correlation function in 460 isosceles configurations smaller than $5^{\circ}$ on a set of 81 discs, after subtraction of all power on

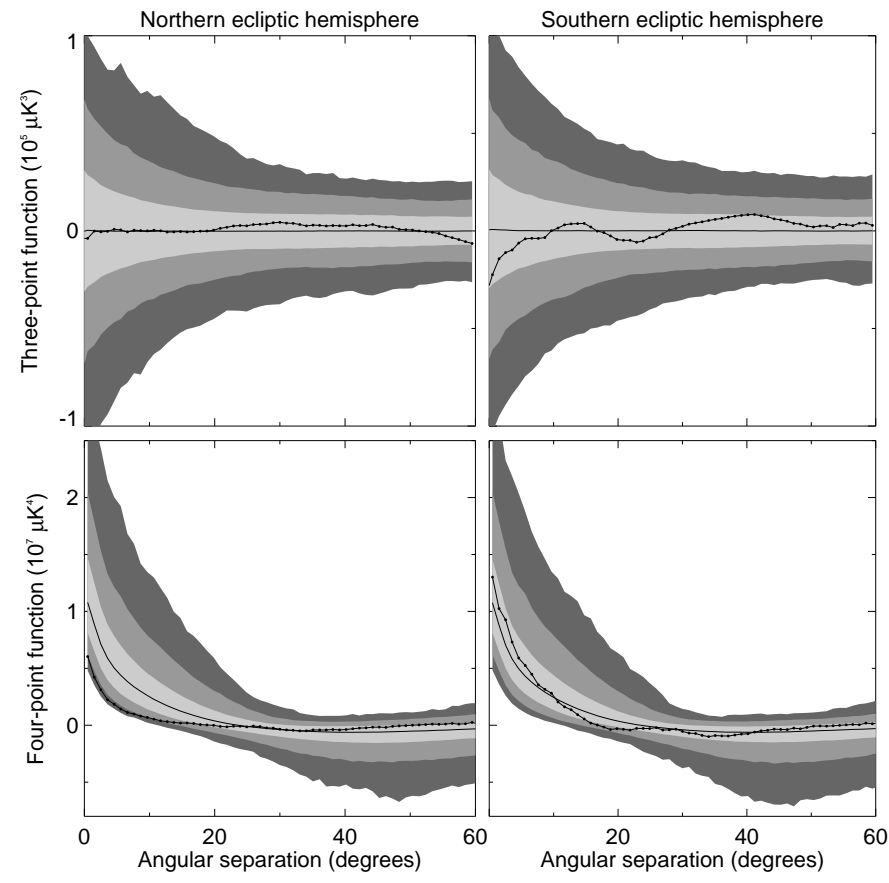

FIG. 3.- Pseudo-collapsed three-point and $1+3$ four-point correlation functions (dotted line) as computed from the low-resolution co-added Q-, V- and W-band WMAP data for the Kp0 Galactic mask after imposing an additional constraint that $|b|>30^{\circ}$. The gray bands indicate 1,2 , and $3 \sigma$ confidence regions as computed from a set of 5000 Monte Carlo simulations. The solid line shows the median.

scales $\ell<18$. The result is shown in the lower panel of Figure 1 to aid comparison with the power spectrum results shown in the middle panel. An asymmetry is also clearly observed on these scales: large connected regions on the northern hemisphere have a low $\chi^{2}$, whereas the southern hemisphere regions (especially in the eastern quadrant) demonstrate higher values. The possible alignment of structure with the ecliptic poles is in very good agreement with both the $N$-point results derived from the ecliptic hemispheres and the power spectrum analysis over the range $\ell=20-40$.

\section{CONCLUSIONS}

In this paper we have estimated power spectra and $N$ point correlation functions from the WMAP first-year Q-, V- and W-band data, and compared these against Monte Carlo ensembles based on Gaussian realizations of the WMAP best-fit running-index power spectrum. Subsequently, we have found evidence for an anisotropic distribution of large-scale power, as implied by the fact that the ratio between the northern and southern ecliptic hemisphere power spectra is low at the $3 \sigma$ level. When the WMAP data are observed in a reference frame that maximizes the asymmetry between hemispheres, the observed ratio determined from the power spectrum over the range $\ell=2-40$ is higher than that computed for at least $99.7 \%$ of the simulations. This is the case even when the asymmetry ratio for each simulation is computed in its own specific reference frame of maximum asymmetry. The significance of the result is therefore independent of the reference-frame. The asymmetry was also confirmed by an $N$-point correlation function analysis, in which the 
ratio of $\chi^{2}$ values computed from the ecliptic hemisphere correlation functions is low, typically at the $98 \%$ level, and in some cases, even as low as $0.1 \%-0.4 \%$.

The origin of the asymmetry is most simply expressed as a remarkable absence of large-scale power in the vicinity of the north ecliptic pole. Nevertheless, it is also a possibility that fundamentally non-Gaussian signals contribute. Indeed, the three-point correlation function in the northern ecliptic hemisphere exhibits what could be described as "super-Gaussianity." Such a property had previously been determined for the $C O B E$-DMR data with a bispectrum statistic (Magueijo 2000). Whether this is the case for WMAP remains to be seen, but such an unexpected lack of deviation from the zero expectation could be interpreted either way.

It is certain that one criticism of this work could be that, given $N$ statistical tests as applied to a specific data set, some may prove to be anomalous simply by chance. The specious nature of this argument should be clear, since the assertion only applies for tests concerning the same non-Gaussian degree of freedom, whereas we have presented (at least) three. Moreover, it is certainly rendered moot by the increasing number of recent supporting results in the literature. In particular, Park (2004) has independently discovered the north-south asymmetry through the use of genus statistics. In addition, the low quadrupole and octopole amplitudes already noted in the $C O B E$-DMR data have been confirmed by the WMAP team itself, but more interestingly, de OliveiraCosta et al. (2004) have noted a conspicuous alignment of these features. Finally, several groups have made claims for the detection of non-Gaussianity with a variety of tests based on phase (Naselsky et al. 2004; Coles et al. 2004), wavelets (Vielva et al. 2004), and multipole vectors (Copi et al. 2004).

That the WMAP data does indeed contain unusual features on large scales seems inescapable. The main issue now is to elucidate possible explanations; three possible candidates are that these effects are due to systematics, foregrounds and exotic physics.
It would appear that the WMAP data are remarkably free of systematic artifacts (Hinshaw et al. 2003a). Moreover, the results presented in this paper seem unlikely to be compromised by systematics since we find supporting evidence from similar features in the $C O B E$ DMR maps, which are susceptible to different parasitic signals. Nevertheless, either previously unknown signals could be postulated, or one might accept the possibility that the projection of the known systematic signals onto these new statistical tests is more complex than expected (cf. the $C O B E$-DMR bispectrum situation as discussed by Banday, Zaroubi, \& Górski 2000).

Residual foreground contamination also seems unlikely. In particular, we note that the $\ell=20-40$ power spectrum results find an axis of maximum asymmetry such that the north pole of the coordinate system lies away from the Galactic plane and the axis separating the hemispheres is highly inclined to it. More importantly, the observed asymmetry is remarkably stable with respect to frequency and sky coverage.

The most intriguing possibility remains therefore that the recent findings require some fundamentally new physics on large scales in the universe.

FKH acknowledges financial support from the CMBNET Research Training Network and HKE and PBL acknowledge financial support from the Research Council of Norway. Some of the results in this paper have been derived using the HEALPix (Górski et al. 1999) software and analysis package. We acknowledge use of the Legacy Archive for Microwave Background Data Analysis (LAMBDA). Support for LAMBDA is provided by the NASA Office of Space Science. This research used resources of the National Energy Research Scientific Computing Center, which is supported by the Office of Science of the U.S. Department of Energy under Contract No. DE-AC03-76SF00098. This work has also received support from The Research Council of Norway (Programme for Supercomputing) through a grant of computing time.

\section{REFERENCES}

Banday, A. J., Zaroubi, S., \& Górski, K. M. 2000, ApJ, 533, 575

Bennett, C. L., et al. 2003a, ApJS, 148, 1

Bennett, C. L., et al. 2003b, ApJS, 148, 97

Coles, P., Dineen, P., Earl, J., \& Wright, D. 2004, MNRAS, submitted (astro-ph/0310252)

Copi, C. J., Huterer, D., \& Starkman, G. D. 2004, preprint (astro-ph/0310511)

de Oliveira-Costa, A., Tegmark, M., Zaldarriaga, M., \& Hamilton, A. 2004, preprint (astro-ph/0307282)

Efstathiou, G. 2003a, MNRAS, 343, L95

Efstathiou, G. 2003b, MNRAS, 346, L26

Efstathiou, G. 2004, MNRAS, submitted (astro-ph/0310207)

Eriksen, H. K., Banday, A. J., \& Górski, K. M. 2002, A\&A, 395, 409

Gaztañaga, E., \& Wagg, J. 2003, Phys. Rev. D, 68, 021302

Górski, K. M., Hivon, E., \& Wandelt, B. D. 1999, in Evolution of Large-Scale Structure: From Recombination to Garching, ed. A. J. Banday, R. K. Sheth, \& L. N. da Costa (Garching: ESO), 37
Hansen, F. K., Górski, K. M., \& Hivon, E. 2002, MNRAS, 336, 1304

Hinshaw, G., et al. 2003a, ApJS, 148, 63

Hinshaw, G., et al. 2003b, ApJS, 148, 135

Magueijo, J. 2000, ApJ, 528, L57

Naselsky, P. D., Verkhodanov, O. V., Chiang, L.-Y., \& Novikov, I. D. 2004, ApJ, submitted (astro-ph/0310235)

Page, L., et al. 2003, ApJS, 148, 39

Park, C.-G. 2004, MNRAS, submitted (astro-ph/0307469)

Spergel, D. N., et al. 2003, ApJS, 148, 175

Tegmark, M., de Oliveira-Costa, A., \& Hamilton, A. 2003, Phys. Rev. D, 68, 123523

Vielva, P., Martinez-Gonzalez E., Barreiro, R. B., Sanz, J. L., \& Cayon, L. 2004, preprint (astro-ph/0310273) 